\title{
Invasive Alien Species of Terrestrial Vegetation of North-Eastern Uttar Pradesh
}

\author{
Sumit Srivastava, Ashish Dvivedi, and Ravindra Prasad Shukla \\ Plant Ecology Laboratory, Department of Botany, DDU, Gorakhpur University, Gorakhpur 273009, India \\ Correspondence should be addressed to Sumit Srivastava; srivastava980@gmail.com
}

Received 19 January 2014; Accepted 21 April 2014; Published 12 May 2014

Academic Editor: Robin Reich

Copyright (C) 2014 Sumit Srivastava et al. This is an open access article distributed under the Creative Commons Attribution License, which permits unrestricted use, distribution, and reproduction in any medium, provided the original work is properly cited.

\begin{abstract}
The vegetational landscape of north-eastern Terai region at the foot hills of Central Himalayas is a mosaic of grassland, old-field, wasteland, and forest ecosystems. Like many other parts of the country, this region is also infested with alien intruders which not only interfere with the growth and production of food crops but also exercise adverse effects on the biodiversity of native species. The present study attempts to catalogue the invasive alien species of the terrestrial vegetation of north-eastern Uttar Pradesh especially with reference to their habit, taxonomic position, and nativity. A total of 1135 plant species within 580 genera under 119 families are so far known to occur in the region. Of these, only 149 species within 100 genera under 41 families have been found to be invasive aliens as evident from their center of origin, past history, nature of aggregation, and invasion observed under field conditions. About $80 \%$ of these invaders have been introduced from neotropics. Out of 173 invasive plants across India, this region shares 149 species, out of which $66 \%$ of species have come from Tropical America, $14 \%$ from African continent, and the rest from other countries. A better planning in the form of early identification and reporting of infestation and spread of noxious weeds is needed for their control.
\end{abstract}

\section{Introduction}

Invasion of alien plant species in recent times has been recognized as the second worst threat after habitat destruction [1]. The International Union for Conservation of Nature and Natural Resources (IUCN) defines "alien invasive species" as an alien species which becomes established in natural or seminatural ecosystems or habitat as agent of change and threatens native biological diversity. Identifying the processes that determine the distribution and diversity of exotic species is a major area of research in ecology because exotics impose significant economic, social, and environmental costs, including human health [2]. Biological invasion may be considered as a form of biological pollution and the significant component of anthropogenic changes leading to extinction of native species. The ecological approach to plant invasion has been mostly based on (a) biological and ecological features promoting the invasion success of particular species $[3,4]$ and (b) the character and invasibility of invaded communities [5]. Recently, both approaches are treated as complementary $[6,7]$. The phytogeographical and floristic approaches are important for research on alien plants [8]. A number of workers have studied and provided catalogues of the invasive alien plant species in different parts of the world [9-12]. Establishment of a database of naturalized species is the first step in the development of invasion biology. A naturalized species is an introduced species that can consistently reproduce and sustain population over many generations without direct intervention by humans $[13,14]$. After successful establishment few naturalized species disperse and produce viable offsprings in areas far from the sites of their introduction. Such naturalized species are then called invasive. It is estimated that as many as $50 \%$ of invasive species, in general, can be classified as ecologically harmful [13].

Due to increasing trade and transcontinental transport, the floras of Indian subcontinent have a number of alien species from various parts of the world as evident from the studies made at different parts in India, namely, Upper Gangetic Plain [15, 16], Khandwa Plateau [17], Kodaikanal 
and Palani Hills [18], Kashmir Himalaya [19, 20], Ranchi [21], Gangtok [22], Allahabad [23], Rajasthan [24], South Gujarat [25], and Doon Valley [26]. This paper presents observations on the habit and nativity of invaders of north-eastern Uttar Pradesh and their impact on the diversity of native plants.

\section{The Site, Climate, and Vegetation}

The study was conducted in the Terai landscape of northeastern Uttar Pradesh. This area is characterized by even topography and fine alluvial deposits from Rapti and Gandak rivers. Mean altitude of the study area is $95 \mathrm{~m}$ amsl. The region slopes gently from north-west to south-east direction. Administratively the study area $\left(\sim 10,000 \mathrm{~km}^{2} ; 27^{\circ} 5^{\prime}\right.$ to $27^{\circ} 40^{\prime} \mathrm{N}$ latitude and $83^{\circ} 30^{\prime}$ to $84^{\circ} \mathrm{E}$ longitude) falls in Gorakhpur district of Uttar Pradesh state. It is bounded by Nepal in the north and Bihar state of India in the east. The landscape comprises a mosaic of human habitations, agricultural fields, grasslands, commercial plantations, and forests. The climate is typically monsoonic with three distinct seasons, namely, summer (March to mid-June), rainy (midJune to mid-October), and winter (mid-October to February). The total average annual rainfall is about $1814 \mathrm{~mm}$; about $87 \%$ of annual rainfall is received during warm rainy season and the rest $13 \%$ is distributed in the form of occasional showers from November to May. Relative humidity ranges between 74 and $87 \%$. The mean maximum temperatures during wet summer, winter, and dry summer season are $35.2^{\circ}$, $27^{\circ}$, and $24-39^{\circ} \mathrm{C}$ and mean minimum temperatures are $26.2^{\circ}$, $12.1^{\circ}$, and $24.2^{\circ} \mathrm{C}$, respectively (based on climatic data for 2000-2005). The soil of the region is classified as Gangetic alluvium, ranging from clayey to sandy loam in texture with $\mathrm{pH}$ ranging from 6.5 to 7.5 . In the northern area there are a few elevated mounds, locally called dhus, which range in size from a few hundred meters to $4-5 \mathrm{~km}$ and have brown sandy soil.

\section{Data Sources and Methods}

Over the period between 1960 and 2013, a comprehensive list of invasive alien plant species of north-eastern Uttar Pradesh was made. The truly aquatic plants were avoided but marshy plants were considered as component of terrestrial vegetation. Several extensive reviews on invasive plant species are available [12, 27-33]. The website http://www.isws.in/invasive-plants-of-india.php [34] was also searched for information on the origin and nativity of these invaders. Some information pertaining to the nativity of the species in India has been extracted from 18, 21-24, and 36-40.

Invasive alien species occurring in this region were compiled based on the literature survey, field observation, and discussion with local people. They were divided into three categories: naturalized, interfering, and noxious. Self-replacing plant populations by recruitment through seeds/ramets and capable of independent growth were categorized as naturalized. Alien and native plants which impacted agriculture adversely especially on the disturbed sites were taken as noxious. The adverse impact of noxious species was in the form of competition for space with tillage or forage crops and harbouring of pests or disease vectors, harmful to crops/native species. In addition to efficient vegetative mode of propagation the seeds of these species are mostly wind-distributed and may remain viable for several years. The species which were neither injurious nor noxious but caused profuse interference and hindrance to the growth of crop/native species over a large area by virtue of their vast numbers were taken as interfering.

\section{Results}

A total of 149 species of invasive aliens of the terrestrial vegetation of north-eastern Uttar Pradesh have been documented. These 149 alien species belonged to 100 genera under 41 families. The alien species amounted to $13.1 \%$ of 1135 wild terrestrial plant species of the region. The habit, nativity, and the impact of invasive species on forest, grassland, and agricultural communities were noticed to prepare a catalogue of invasive alien/exotic plant species (Table 1). 100 aliens have their origin in Tropical America compared to 21 species in African continent. About 28 species of alien plants reached the study area from such far off places as Afghanistan, Australia, Brazil, East Indies, Europe, Madagascar, Mascarene Islands, Mediterranean, Mexico, Peru, Temperate South America, Tropical West Asia, West Indies, and Western Europe.

The herbaceous elements predominated the regional alien flora. The number of dicot alien species was 136, under 93 genera and 32 families. On the other hand, there were only 13 species of monocot aliens distributed among 9 genera under 5 families (Amaryllidaceae, Arecaceae, Cyperaceae, Liliaceae, and Poaceae). Of 41 families having alien species, Asteraceae was the most dominant (29 species) followed by Convolvulaceae (11), Amaranthaceae and Solanaceae (9 species each), Euphorbiaceae (8), Caesalpiniaceae and Papilionaceae (7 each), Poaceae (6), Capparidaceae, Cyperaceae, Tiliaceae, and Verbenaceae (4 each), Asclepiadaceae, Chenopodiaceae, Lamiaceae, Malvaceae, Mimosaceae, and Onagraceae (3 each), Acanthaceae, Oxalidaceae, Papaveraceae, Pedaliaceae, Portulacaceae, and Scrophulariaceae (2 each), and Amaryllidaceae, Apocynaceae, Arecaceae, Brassicaceae, Cannabaceae, Cuscutaceae, Cactaceae, Liliaceae, Polygonaceae, Primulaceae, Passifloraceae, Piperaceae, Rosaceae, Sterculiaceae, Ulmaceae, Urticaceae, and Zygophyllaceae (1 species each). Of these aliens, 15 species were judged as noxious, 43 species as interfering, and 91 as naturalized species (Figure 1). Habit wise analysis shows that $80 \%$ of species are herbs, $12 \%$ are shrubs, $6 \%$ are herbaceous climbers, and $2 \%$ are trees (Figure 2 ). The eight dominant families contributed $47 \%$ of the invasive alien flora of wild terrestrial vegetation of north-eastern Uttar Pradesh (Figure 3).

\section{Discussion}

Alien species are nonnative or exotic organisms that occur outside their natural adapted ranges and dispersal potential 
TABLE 1: Wild invasive plant species in terrestrial vegetation of north-eastern Uttar Pradesh.

\begin{tabular}{|c|c|c|c|c|c|}
\hline S. No. & Name of the Species & Family & Habit & Nativity & Categories \\
\hline 1 & Acacia farnesiana (L.) Willd & Mimosaceae & Tree & Tropical South America & Naturalized \\
\hline 2 & Acanthospermum hispidum DC. & Asteraceae & Herb & Brazil & Naturalized \\
\hline 3 & Aerva tomentosa & Amaranthaceae & Herb & Tropical America & Naturalized \\
\hline 4 & Ageratum conyzoides $\mathrm{L}$. & Asteraceae & Herb & Tropical America & Noxious \\
\hline 5 & Ageratum houstonianum Mill. & Asteraceae & Herb & Tropical America & Interfering \\
\hline 6 & Alternanthera paronychioides A. St. Hill & Amaranthaceae & Herb & Tropical America & Naturalized \\
\hline 7 & Alternanthera pungens Kunth & Amaranthaceae & Herb & Tropical America & Naturalized \\
\hline 8 & Alternanthera sessilis (L.) R. Br. ex DC. & Amaranthaceae & Herb & Tropical America & Naturalized \\
\hline 9 & Amaranthus spinosus L. & Amaranthaceae & Herb & Tropical America & Naturalized \\
\hline 10 & Anagallis arvensis $\mathrm{L}$. & Primulaceae & Herb & Europe & Naturalized \\
\hline 11 & Antigonon leptopus Hook and Arn. & Polygonaceae & climber & Tropical America & Noxious \\
\hline 12 & Argemone mexicana L. & Papaveraceae & Herb & Tropical South America & Noxious \\
\hline 13 & Argemone ochroleuca Sweet. & Papaveraceae & Herb & Mexico & Interfering \\
\hline 14 & Asphodelus tenuifolius Cav. & Liliaceae & Herb & Tropical America & Naturalized \\
\hline 15 & Blainvillea acmella (L.) Philipson & Asteraceae & Herb & Tropical America & Interfering \\
\hline 16 & Blumea eriantha DC. & Asteraceae & Herb & Tropical America & Interfering \\
\hline 17 & Blumea lacera (Burm f.) DC. & Asteraceae & Herb & Tropical America & Interfering \\
\hline 18 & Blumea obliqua (L.) Druce & Asteraceae & Herb & Tropical America & Interfering \\
\hline 19 & Borassus flabellifer $\mathrm{L}$. & Arecaceae & Tree & Tropical Africa & Naturalized \\
\hline 20 & Calotropis gigantea (L.) R. Br. & Asclepiadaceae & Shrub & Tropical Africa & Interfering \\
\hline 21 & Calotropis procera (Ait) R. Br. & Asclepiadaceae & Shrub & Tropical Africa & Interfering \\
\hline 22 & Cannabis sativa $\mathrm{L}$. & Cannabaceae & Herb & Central Asia & Interfering \\
\hline 23 & Cassia absus L. & Caesalpiniaceae & Herb & Tropical America & Naturalized \\
\hline 24 & Cassia alata $\mathrm{L}$. & Caesalpiniaceae & Shrub & West Indies & Naturalized \\
\hline 25 & Cassia hirsute L. & Caesalpiniaceae & Herb & Tropical America & Naturalized \\
\hline 26 & Cassia obtusifolia L. & Caesalpiniaceae & Herb & Tropical America & Naturalized \\
\hline 27 & Cassia occidentalis L. & Caesalpiniaceae & Herb & Tropical South America & Naturalized \\
\hline 28 & Cassia pumila Lam. & Caesalpiniaceae & Herb & Tropical America & Naturalized \\
\hline 29 & Cassia tora $\mathrm{L}$. & Caesalpiniaceae & Herb & Tropical South America & Noxious \\
\hline 30 & Celosia argentea $\mathrm{L}$. & Amaranthaceae & Herb & Tropical Africa & Naturalized \\
\hline 31 & Chenopodium album $\mathrm{L}$. & Chenopodiaceae & Herb & Europe & Interfering \\
\hline 32 & Chenopodium ambrosioides $\mathrm{L}$. & Chenopodiaceae & Herb & Tropical America & Interfering \\
\hline 33 & Chenopodium murale L. & Chenopodiaceae & Herb & Tropical America & Naturalized \\
\hline 34 & Chloris barbata Sw. & Poaceae & Herb & Tropical America & Naturalized \\
\hline 35 & Chrozophora rottleri (Geis) Spring & Euphorbiaceae & Herb & Tropical Africa & Naturalized \\
\hline 36 & Cleome burmanni & Capparidaceae & Herb & West Africa & Naturalized \\
\hline 37 & Cleome gynandra L. & Capparidaceae & Herb & Tropical America & Naturalized \\
\hline 38 & Cleome rutidosperma DC. & Capparidaceae & Herb & Tropical America & Naturalized \\
\hline 39 & Cleome viscosa $\mathrm{L}$. & Capparidaceae & Herb & Tropical America & Naturalized \\
\hline 40 & Clerodendrum splendens G. Don & Verbenaceae & Climber & Africa & Interfering \\
\hline 41 & Convolvulus arvensis $\mathrm{L}$. & Convolvulaceae & Herb & Europe & Naturalized \\
\hline 42 & Corchorus aestuans L. & Tiliaceae & Herb & Tropical America & Naturalized \\
\hline 43 & Corchorus fascicularis Lam. & Tiliaceae & Herb & Tropical America & Naturalized \\
\hline 44 & Corchorus olitorius L. & Tiliaceae & Herb & Tropical Africa & Naturalized \\
\hline 45 & Coronopus didymus (L.) Smith & Brassicaceae & Herb & Tropical America & Interfering \\
\hline 46 & Crotalaria mucronata.Ait & Papilionaceae & Herb & Tropical America & Interfering \\
\hline 47 & Croton bonplandianum Boil. & Euphorbiaceae & Herb & Temperate South America & Naturalized \\
\hline 48 & Cryptostegia grandiflora R. Br. & Asclepiadaceae & Herb & Madagascar & Interfering \\
\hline
\end{tabular}


TABLe 1: Continued.

\begin{tabular}{|c|c|c|c|c|c|}
\hline S. No. & Name of the Species & Family & Habit & Nativity & Categories \\
\hline 49 & Cuscuta reflexa Roxb. & Cuscutaceae & Herb & Mediterranean & Interfering \\
\hline 50 & Cynodon dactylon (Linn.) Pers & Poaceae & Herb & Africa & Naturalized \\
\hline 51 & Cyperus difformis $\mathrm{L}$. & Cyperaceae & Herb & Tropical America & Naturalized \\
\hline 52 & Cyperus flabelliformis Rottb. & Cyperaceae & Herb & Africa & Interfering \\
\hline 53 & Cyperus iria L. & Cyperaceae & Herb & Tropical America & Naturalized \\
\hline 54 & Cyperus cyperoides L. & Cyperaceae & Herb & Tropical America & Naturalized \\
\hline 55 & Datura innoxia Mill. & Solanaceae & Shrub & Tropical America & Noxious \\
\hline 56 & Datura metel L. & Solanaceae & Shrub & Tropical America & Interfering \\
\hline 57 & Datura stramonium L. & Solanaceae & Shrub & Tropical America & Noxious \\
\hline 58 & Digera muricata (L.) Mart & Amaranthaceae & Herb & South-West Asia & Interfering \\
\hline 59 & Duranta repens Linn. & Verbenaceae & Shrub & America & Naturalized \\
\hline 60 & Echinochloa colonum (L.) Link & Poaceae & Herb & Tropical South America & Naturalized \\
\hline 61 & Echinochloa crus-galli (L.) Beauv. & Poaceae & Herb & Tropical South America & Noxious \\
\hline 62 & Echinops echinatus Roxb. & Asteraceae & Herb & Afghanistan & Naturalized \\
\hline 63 & Eclipta prostrata (L.) Mart. & Asteraceae & Herb & Tropical America & Naturalized \\
\hline 64 & Emilia sonchifolia (L.) DC. & Asteraceae & Herb & Tropical America & Naturalized \\
\hline 65 & Erigeron bonariensis L. & Asteraceae & Herb & South America & Interfering \\
\hline 66 & Eupatorium adenophorum Sprengel & Asteraceae & Shrub & Mexico & Noxious \\
\hline 67 & Eupatorium odoratum L. & Asteraceae & Shrub & Tropical America & Interfering \\
\hline 68 & Euphorbia chamaesyce L. & Euphorbiaceae & Herb & West Africa \& Mauritius & Naturalized \\
\hline 69 & Euphorbia hirta L & Euphorbiaceae & Herb & Tropical America & Naturalized \\
\hline 70 & Euphorbia heterophylla L. & Euphorbiaceae & Herb & Tropical America & Naturalized \\
\hline 71 & Evolvulus nummularius (L.) L. & Convolvulaceae & Herb & Tropical America & Naturalized \\
\hline 72 & Flaveria trinervia (Spreng.) C. Mohar & Asteraceae & Herb & Tropical Cent. America & Naturalized \\
\hline 73 & Galinsoga parviflora Cav. & Asteraceae & Herb & Tropical America & Naturalized \\
\hline 74 & Glossocardia bosvallia (L. f.) DC. & Asteraceae & Herb & West Indies & Naturalized \\
\hline 75 & Gnaphalium polycaulon Pers. & Asteraceae & Herb & Tropical America & Interfering \\
\hline 76 & Gomphrena celosioides Mart. & Amaranthaceae & Herb & Tropical America & Naturalized \\
\hline 77 & Gomphrena globosa Linn. & Amaranthaceae & Herb & America & Naturalized \\
\hline 78 & Grangea maderaspatana (L.) Poir. & Asteraceae & Herb & Tropical South America & Naturalized \\
\hline 79 & Hyptis suaveolens (L.) Poit & Lamiaceae & Herb & Tropical America & Interfering \\
\hline 80 & Imperata cylindrica (L.) Raensch. & Poaceae & Herb & Tropical America & Naturalized \\
\hline 81 & Indigofera glandulosa Roxb. Ex Willd & Papilionaceae & Herb & Tropical America & Naturalized \\
\hline 82 & Indigofera hirsuta Hook. & Papilionaceae & Herb & Tropical Africa & Naturalized \\
\hline 83 & Indigofera linearis Ali & Papilionaceae & Herb & Tropical Africa & Naturalized \\
\hline 84 & Indigofera linifolia (L. f.) Retz. & Papilionaceae & Herb & Tropical South America & Naturalized \\
\hline 85 & Ipomoea eriocarpa $\mathrm{R}$. Br. & Convolvulaceae & Climber & Tropical Africa & Interfering \\
\hline 86 & Ipomoea fistulosa Mart DC. & Convolvulaceae & Shrub & Tropical America & Interfering \\
\hline 87 & Ipomoea muricata $\mathrm{L}$. & Convolvulaceae & Climber & Tropical America & Naturalized \\
\hline 88 & Ipomoea nil (L.) Roth. & Convolvulaceae & Climber & North America & Naturalized \\
\hline 89 & Ipomoea obscura (L.) Ker. Gawl. & Convolvulaceae & Climber & Tropical Africa & Interfering \\
\hline 90 & Ipomoea pes-tigridis L. & Convolvulaceae & Climber & Tropical East Africa & Interfering \\
\hline 91 & Ipomoea purpurea (Linn.) Roth & Convolvulaceae & Herb & America & Interfering \\
\hline 92 & Ipomoea quamoclit $\mathrm{L}$. & Convolvulaceae & Climber & Tropical America & Interfering \\
\hline 93 & Jatropha curcas & Euphorbiaceae & Herb & Tropical America & Naturalized \\
\hline 94 & Jatropha gossypifolia L. & Euphorbiaceae & Shrub & Brazil & Naturalized \\
\hline 95 & Lagascea mollis Cav & Asteraceae & Herb & Tropical Cent. America & Noxious \\
\hline 96 & Lantana camara L. & Verbenaceae & Shrub & Tropical America & Noxious \\
\hline 97 & Leonotis nepetifolia (L.) R. Br. & Lamiaceae & Herb & Tropical Africa & Interfering \\
\hline
\end{tabular}


TABle 1: Continued.

\begin{tabular}{|c|c|c|c|c|c|}
\hline S. No. & Name of the Species & Family & Habit & Nativity & Categories \\
\hline 98 & Leucaena leucocephala (Lam.) de. Wit & Mimosaceae & Herb & Tropical America & Noxious \\
\hline 99 & Lippia alba & Verbenaceae & Herb & Trop. America \& West Indies & Interfering \\
\hline 100 & Ludwigia adscendens (L.) Hara & Onagraceae & Herb & Tropical America & Naturalized \\
\hline 101 & Ludwigia octovalvis (Jacq.) Raven & Onagraceae & Herb & Tropical America & Naturalized \\
\hline 102 & Ludwigia parviflora Roxb. & Onagraceae & Herb & Tropical Africa & Naturalized \\
\hline 103 & Malvastrum coromandelianum (L.) Gar. & Malvaceae & Herb & Tropical America & Naturalized \\
\hline 104 & Martynia annua L. & Pedaliaceae & Herb & Tropical America & Interfering \\
\hline 105 & Mecardonia procumbens (Mill.) Small & Scrophulariaceae & Herb & Tropical North America & Naturalized \\
\hline 106 & Melilotus alba Desv. & Papilionaceae & Herb & Europe & Naturalized \\
\hline 107 & Melochia corchorifolia $\mathrm{L}$. & Sterculiaceae & Herb & Tropical America & Naturalized \\
\hline 108 & Merremia dissecta (Jacq.) Hallier $\mathrm{f}$. & Convolvulaceae & Herb & Tropical America & Naturalized \\
\hline 109 & Mikania micrantha Kunth. & Asteraceae & Climber & Tropical America & Noxious \\
\hline 110 & Mimosa pudica L. & Mimosaceae & Herb & Brazil & Naturalized \\
\hline 111 & Nicotiana plumbaginifolia Viv. & Solanaceae & Herb & Tropical America & Naturalized \\
\hline 112 & Ocimum americanum $\mathrm{L}$. & Lamiaceae & Herb & Tropical America & Naturalized \\
\hline 113 & Opuntia elatior Mill. & Cactaceae & Herb & Tropical America & Noxious \\
\hline 114 & Oxalis corniculata $\mathrm{L}$. & Oxalidaceae & Herb & Europe & Naturalized \\
\hline 115 & Oxalis corymbosa DC. & Oxalidaceae & Herb & South America & Naturalized \\
\hline 116 & Parthenium hysterophorus $\mathrm{L}$ & Asteraceae & Herb & Tropical North America & Noxious \\
\hline 117 & Passiflora foetida $\mathrm{L}$. & Passifloraceae & Herb & Tropical South America & Interfering \\
\hline 118 & Pedalium murex $\mathrm{L}$. & Pedaliaceae & Herb & Tropical America & Naturalized \\
\hline 119 & Peperomia pellucida (L.) Kunth. & Piperaceae & Herb & Tropical South America & Naturalized \\
\hline 120 & Peristrophe paniculata (Forsk.) Brummitt & Acanthaceae & Herb & Tropical America & Interfering \\
\hline 121 & Physalis minima L. & Solanaceae & Herb & Tropical America & Naturalized \\
\hline 122 & Physalis peruviana $\mathrm{L}$ & Solanaceae & Herb & Tropical America & Interfering \\
\hline 123 & Pilea microphylla & Urticaceae & Herb & Tropical South America & Naturalized \\
\hline 124 & Portulaca oleracea L. & Portulacaceae & Herb & Tropical S. America & Naturalized \\
\hline 125 & Portulaca quadrifida $\mathrm{L}$. & Portulacaceae & Herb & Tropical America & Naturalized \\
\hline 126 & Rauvolfia tetraphylla & Apocynaceae & Herb & West Indies & Naturalized \\
\hline 127 & Ricinus communis Linn. & Euphorbiaceae & Shrub & Africa & Interfering \\
\hline 128 & Rubus ellipticus Smith & Rosaceae & Shrub & Tropical America & Naturalized \\
\hline 129 & Ruellia tuberosa L. & Acanthaceae & Herb & Tropical America & Naturalized \\
\hline 130 & Saccharum spontaneum L. & Poaceae & Herb & Tropical West Asia & Interfering \\
\hline 131 & Scoparia dulcis $\mathrm{L}$. & Scrophulariaceae & Herb & Tropical America & Naturalized \\
\hline 132 & Sesbania bispinosa (Jacq.) W. F. Wight & Papilionaceae & Shrub & Tropical America & Naturalized \\
\hline 133 & Sida acuta Burm f. & Malvaceae & Herb & Tropical America & Naturalized \\
\hline 134 & Solanum khasianum & Solanaceae & Herb & Tropical America & Interfering \\
\hline 135 & Solanum nigrum $\mathrm{L}$. & Solanaceae & Herb & Tropical America & Naturalized \\
\hline 136 & Solanum torvum Sw. & Solanaceae & Shrub & West Indies & Interfering \\
\hline 137 & Solvia anthemifolia (Juss) R. & Asteraceae & Herb & America & Interfering \\
\hline 138 & Sonchus asper Hill. & Asteraceae & Herb & Mediterranean & Interfering \\
\hline 139 & Sonchus oleraceus L. & Asteraceae & Herb & Mediterranean & Interfering \\
\hline 140 & Synedrella nodiflora (L.) Gaertn. & Asteraceae & Herb & West Indies & Naturalized \\
\hline 141 & Tithonia diversifolia (Hense) A. Gray & Asteraceae & Shrub & Mexico & Naturalized \\
\hline 142 & Trema orientalis L. & Ulmaceae & Tree & Africa & Naturalized \\
\hline 143 & Tribulus terrestris L. & Zygophyllaceae & Herb & Tropical America & Naturalized \\
\hline 144 & Tridax procumbens L. & Asteraceae & Herb & Tropical Cent. America & Naturalized \\
\hline
\end{tabular}


TABle 1: Continued.

\begin{tabular}{|c|c|c|c|c|c|}
\hline S. No. & Name of the Species & Family & Habit & Nativity & Categories \\
\hline 145 & Triumfetta rhomboidea Jacq. & Tiliaceae & Herb & Tropical America & Naturalized \\
\hline 146 & Urena lobata $\mathrm{L}$. & Malvaceae & Shrub & Tropical Africa & Interfering \\
\hline 147 & Xanthium strumarium L. & Asteraceae & Herb & Tropical America & Noxious \\
\hline 148 & Youngia japonica (L.) DC. & Asteraceae & Herb & Tropical South America & Naturalized \\
\hline 149 & Zephyranthes candida Lindl. & Amaryllidaceae & Herb & America & Naturalized \\
\hline
\end{tabular}

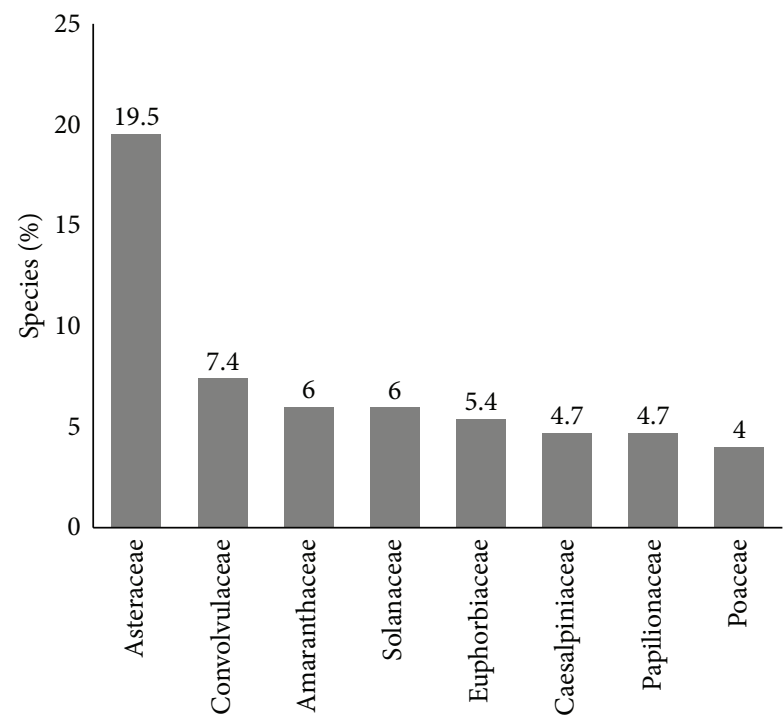

FIGURE 1: Families accounting for $>5$ speciesinvasive in the terrestrial vegetation of north-eastern Uttar Pradesh.

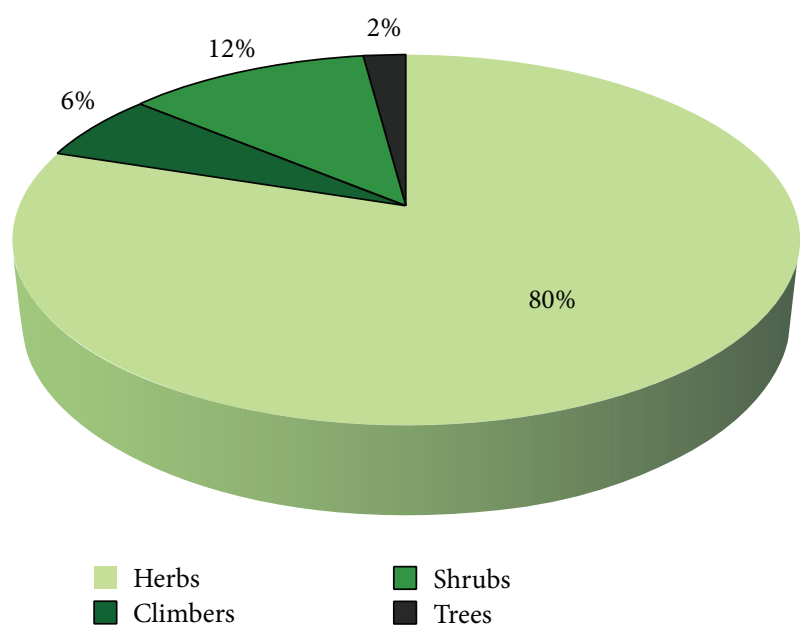

Figure 2: Number (\%) of plant invasive species under different habit categories within terrestrial vegetation of north-eastern Uttar Pradesh.

[35]. These invasive species are widely distributed in all kinds of ecosystems throughout the world and include all categories of living organisms. Nevertheless, plants, mammals, and insects comprise the most common types of invasive alien species in terrestrial environments [36]. Many alien plant species support our farming and forestry systems in a big

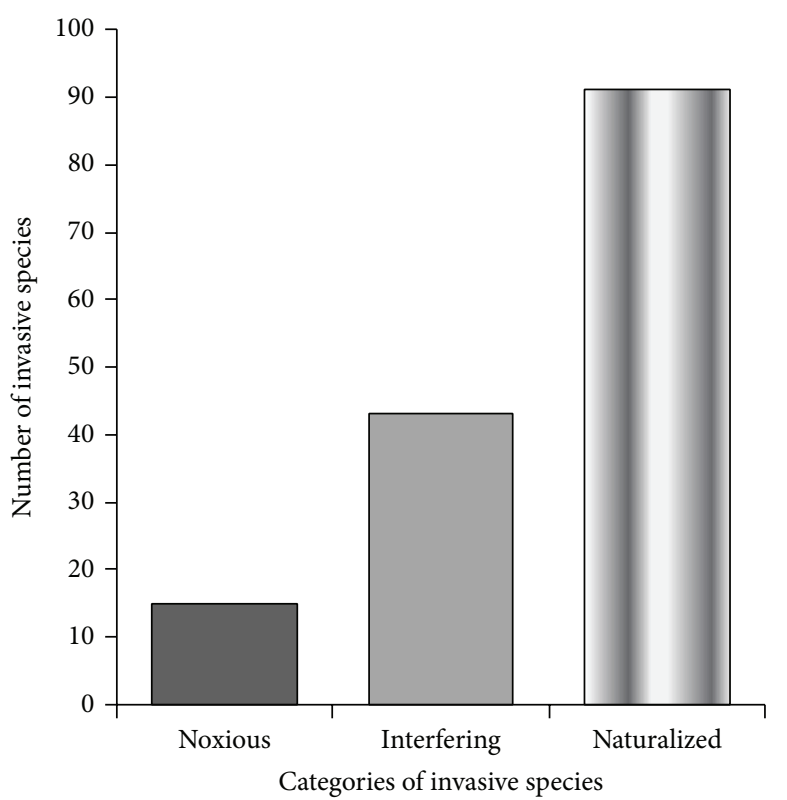

FIGURE 3: Species number under three different categories of invasive plants.

way. However, some of these aliens become invasive when they are introduced deliberately or unintentionally outside their natural habitats into new areas where they express the capability to establish, invade, and outcompete native species [37]. An important requirement for successful colonization of invaders is open habitat with reduced competition. Generally, the microsites created by grazing may be occupied by invader species [38-40]. The invaders usually dominate the highly disturbed and man-made landscapes. So far, no ready hand catalogue of invasive species is available for this region. The present catalogue of invasive exotic species is likely to serve as basic information for future research towards conservation of native plant species of the region.

As evident from the data, the vegetation of northeastern Uttar Pradesh shows greater incidence of invaders as compared to the whole of Uttar Pradesh [41] and India [42]. Reddy documented all invasive plant species irrespective of their habitat and use. In the present case, however, only the wild invasive plant species of terrestrial vegetation were considered and truly aquatic and ornamental invasive species were excluded. Many species, recorded as invader of northeastern Uttar Pradesh, are common to Indian Himalayan region and the whole of Uttar Pradesh. For example, 103 invaders are common to the whole of the state of Uttar 
Pradesh [41] and 95 species to the whole of India [42]. Among the invasive species of north-eastern Uttar Pradesh, 70.5\% are native to American continent. Other such studies vary slightly in percent share of tropical American nativity. While Singh et al. [41] reported 73\% of invasive plant species of Uttar Pradesh, for Indian Himalayan region, however, Sekar [43] also noticed $73 \%$ invaders of American nativity. Reddy [42] noticed $58 \%$ of the invasive flora of India to be natives of American continent.

Alien species have been classified into naturalized and noxious species by various workers [13, 44, 45]. Our field observation and discussion with local people indicate that there are 15 most noxious invasive plant species in this region, namely, Ageratum conyzoides, Antigonon leptopus, Argemone mexicana, Cassia tora, Datura stramonium, Datura innoxia, Echinochloa crus-galli, Eupatorium adenophorum, Lantana camara, Lagascea mollis, Leucaena leucocephala, Mikania micrantha, Parthenium hysterophorus, Opuntia elatior, and Xanthium strumarium. Some species such as Ageratum conyzoides, Eupatorium adenophorum, Lantana camara, and Parthenium hysterophorus are harmful to native species [41, $46,47]$. Further, some of these species are known to be highly allergic, causing diseases in human beings $[48,49]$. Since they are rarely palatable, their dominance drastically reduces the number of grazers by way of reducing the carrying capacity of the pasture and wasteland [40]. Datura innoxia and Datura stramonium are serious threat to the native species of the region and are known to cause delay in seedling growth of neighbouring plants [50]. Leucaena leucocephala alters the natural growth of native plants because not only it obstructs plenty of sunlight to reach surface layer but also its allelopathic exudates cause retardation in seedling growth of neighbouring plants [51]. Mikania micrantha could climb trees and cover the whole habitats through fast vegetative propagation and could also suppress the growth of crops and natural vegetation through competition and allelopathic effects [45]. These species occur as invasive species under shifting agriculture in north-eastern Uttar Pradesh and combine an effective seed based reproduction with clonal propagation [52]. Many invasive species such as species of Eupatorium tend to respond to temporarily nutrientenriched soil substrata and grow and quickly cover the gaps in disturbed forests. They can destroy arable soil, negatively affect the growth of orchard, and could also supplant grasses in pasture, excreting a toxic volatile that prevents grazing [48].

The herbaceous invasive plant species were recorded as the dominant invasive flora $(80 \%)$ of north-east Uttar Pradesh, in the form of 119 invasive species. The greater viability and tolerance to harsh conditions could result in the preponderance of herbs across the region. Invasive species of Asteraceae exhibited a much higher reproductive capacity than those of other families. This high reproductive potential is achieved by partitioning of reproductive capital into a large number of propagules that are minute, light, and wind dispersed [53]. Various other workers have also reported the dominance of Asteraceae among invasive alien species in Uttar Pradesh [41] and in Indian Himalayan region [43].
The invasive species cause loss of biodiversity through species extinction and their impact on ecosystem function. Differences between native and invasive plant species in their resource acquisition and consumption may cause a change in soil structure, decomposition, and nutrient content of the soil. Thus, invasive species are a serious hindrance to conservation with significant undesirable impacts on the goods and services provided by ecosystems. Biological invasions now operate on a global scale and are likely to undergo rapid increase in this century due to interaction with other changes such as increasing travel and tourism. A quick monitoring of invasion can, therefore, be done through qualitative approach like species inventory method.

\section{Conclusion}

Plant invasions in the new areas alter indigenous community composition, deplete species diversity, affect ecosystem process, and thus cause huge economic and ecological imbalance. A quick inventory and plant identification network are, therefore, needed for early detection and reporting of noxious and naturalized weeds in order to control the spread of invasive plant species.

\section{Conflict of Interests}

The authors declare that there is no conflict of interests regarding the publication of this paper.

\section{Acknowledgments}

The authors are thankful to the Head Department of Botany, D.D.U., Gorakhpur University, Gorakhpur, for providing access to departmental herbarium and other required facilities. They also feel grateful to the anonymous reviewer for his critical comments and suggestions to improve the quality and clarity of the content and to Dr. Robin Reich for very quick response.

\section{References}

[1] C . B. D, "Invasive Alien species," Convention on Biological Diversity, 2005, http://www.biodiv.org/programmes/cross-cuttings/Alien.

[2] D. S. Wilcove, D. Rothstein, J. Dubow, A. Phillips, and E. Losos, "Quantifying threats to imperiled species in the U. S.," BioScience, vol. 48, no. 8, pp. 607-615, 1998.

[3] A. E. Newsome and I. R. Noble, "Ecological and physiological characters of invading species," in Ecology of Biological Invasions: An Australian Perspective, R. H. Groves and J. J. Burdon, Eds., pp. 1-20, Australian Academy of Sciences, Canberra, Australia, 1986.

[4] M. Rejmánek, "What makes a species invasive?" in Plant Invasions: General Aspects and Special Problems, P. Pysek, K. Prach, M. Rejmánek, and M. Wade, Eds., pp. 3-13, SPB Academic, Amsterdam, The Netherlands, 1995.

[5] M. Rejmánek, "Invasibility of plant communities," in Biological Invasions: A Global Perspective, J. A. Drake, H. A. Mooney, F. di 
Castri et al., Eds., pp. 369-388, John Wiley \& Sons, Chichester, UK, 1989.

[6] D. M. Lodge, "Biological invasions: lessons for ecology," Trends in Ecology and Evolution, vol. 8, no. 4, pp. 133-137, 1993.

[7] R. J. Hobbs and L. F. Huenneke, "Disturbance, diversity, and invasion: implications for conservation," Conservation Biology, vol. 6, no. 3, pp. 324-337, 1992.

[8] J. A. McNeely, H. A. Mooney, L. E. Neville, P. Schei, and J. K. Waage, "A global strategy on invasive Alien species," in Collaboration With the Global Invasive Species Programme, IUCN, Gland, Switzerland, 2001.

[9] J. A. Drake, H. A. Mooney, F. di Castri et al., Biological Invasions: A Global Perspective, John Wiley \& Sons, New York, NY, USA, 1989.

[10] M. Williamson, Biological Invasions, Chapman \& Hall, New York, NY, USA, 1996.

[11] J. R. Carey, P. Moyle, M. Rejmánek, and G. Vermeij, "Invasion biology," Biological Conservation, vol. 78, pp. 1-214, 1996.

[12] P. Pysek, M. Chytry, J. Pergll, J. Sadloland, and J. Wild, "Plant invasions in the Czech Republic: current state, introduction dynamics, invasive species and invaded habitats," Preslia, vol. 84, pp. 575-629, 2012.

[13] D. M. Richardson, P. Pyšek, M. Rejmánek, M. G. Barbour, F. Dane Panetta, and C. J. West, "Naturalization and invasion of Alien plants: concepts and definitions," Diversity and Distributions, vol. 6, no. 2, pp. 93-107, 2000.

[14] P. Pyšek, J. Sádlo, and B. Mandák, "Catalogue of Alien plants of the Czech Republic," Preslia, vol. 74, no. 2, pp. 97-186, 2002.

[15] M. B. Raizada, "Recently introduced or otherwise imperfectly known plants from the upper gangetic plain," Journal of Indian Botanical Society, vol. 14, pp. 339-348, 1935.

[16] M. B. Raizada, "Recently introduced or otherwise imperfectly known plants from the upper gangetic plain," Journal of Indian Botanical Society, vol. 15, pp. 149-167, 1936.

[17] J. K. Maheshwari, "Studies on the naturalized Flora of India," in Proceedings of the Summer School of Botany, pp. 156-170, New Delhi, India.

[18] K. M. Matthew, "Alien flora of Kodai Kanal and Palni Hills," Records of Botanical Survey of India, vol. 20, pp. 1-241, 1969.

[19] G. Singh and B. Misri, "Some exotic ornamentals of Kashmir," Indian Journal of Horticulture, vol. 31, p. 834, 1974.

[20] G. Singh and P. Kachroo, "Exotic trees and shrubs of Kashmir," Indian Forester, vol. 109, pp. 60-76, 1983.

[21] J. K. Maheswari and S. R. Paul, "The Alien flora of Ranchi," Journal of the Bombay Natural History Society, vol. 72, pp. 158$188,1975$.

[22] P. K. Hajra and B. K. Das, "Vegetation of gangtok with special reference to Alien plants," India Forums, vol. 107, pp. 554-566, 1982.

[23] B. D. Sharma, Exotic Flora of Allahabad, Botanical Survey of India, Dehra Dun, India, 1984.

[24] R. P. Pandey and P. J. Parmar, "The exotic flora of Rajasthan," Journal of Economic and Taxonomic Botany, vol. 18, pp. 105-121, 1994.

[25] S. R. Kshirsagar, "Origin, present status and distribution of exotic plants in South Gujarat," Indian Journal of Forestry, vol. 28, pp. 136-143, 2005.

[26] P. S. Negi and P. K. Hajra, "Alien flora of Doon Valley, Northwest Himalaya," Current Science, vol. 92, no. 7, pp. 968-978, 2007.
[27] H. A. Mooney and J. A. Drake, "The ecology of biological invasions," Environment, vol. 29, no. 5, pp. 10-37, 1987.

[28] C. M. D’Antonio and P. M. Vitousek, "Biological invasions by exotic grasses, the grass/fire cycle, and global change," Annual Review of Ecology and Systematics, vol. 23, no. 1, pp. 63-87, 1992.

[29] P. T. Jenkins, "Trade and exotic species introductions," in Invasive Species and Biodiversity Management, O. T. Sandlund and P. Schei, Eds., pp. 229-235, Kluwer Academic, Dodrecht, The Netherlands, 1999.

[30] H. A. Mooney and R. J. Hobbs, Invasive Species in a Changing World, Island Press, Washington, DC, USA, 2000.

[31] C. S. Elton, The Ecology of Invasions by Animals and Plants, University of Chicago Press, Chicago, Ill, USA, 2000.

[32] R. Cowie, "Does the Public care about Species loss? A Glimpse into the Public's thinking," Conservation Biology in Practice, vol. 2, pp. 28-29, 2001.

[33] E. Wasson, Complete Encyclopedia of Trees and Shrubs: Descriptions, Cultivation Requirements, Pruning, Planting, Thunder Bay Press, San Diego, Calif, USA, 2003.

[34] C. S. Reddy, G. Bagyanarayana, K. N. Reddy, and V. S. Raju, "Invasive Alien Flora of India," National Biological Information Infrastructure, USGS, USA, 2008, http://www.isws.in/invasive-plants-of-india.php.

[35] M. A. McGeoch, S. H. M. Butchart, D. Spear et al., "Global indicators of biological invasion: species numbers, biodiversity impact and policy responses," Diversity and Distributions, vol. 16, no. 1, pp. 95-108, 2010.

[36] A. S. Raghubanshi, L. C. Rai, J. P. Gaur, and J. S. Singh, "Invasive Alien species and biodiversity in India," Current Science, vol. 88, no. 4, pp. 539-540, 2005.

[37] Y. H. Sujay, H. N. Sattagi, and R. K. Patil, "Invasive Alien insects and their impact on agroecosystem," Karnataka Journal of Agricultural Sciences, vol. 23, pp. 26-34, 2010.

[38] P. Singh, "Lantana Weed and Lantana Lace bug," Indian Forester, vol. 102, pp. 474-478, 1976.

[39] P. M. Sinha, "Studies on the use of some Weedicides on Lantana camara," Indian Forester, vol. 102, pp. 298-305, 1976.

[40] V. B. Sawarker, "Lantana camara on wildlife habitats with special reference to the melaghat tiger reserve," Cheetal, vol. 26, pp. 24-38, 1984.

[41] K. P. Singh, A. N. Shukla, and J. S. Singh, "State-level inventory of invasive Alien plants, their source regions and use potential," Current Science, vol. 99, no. 1, pp. 107-114, 2010.

[42] C. S. Reddy, "Catalogue of invasive Alien flora of India," Life Science Journal, vol. 5, no. 2, pp. 84-89, 2008.

[43] K. C. Sekar, "Invasive Alien plants of Indian Himalayan region-diversity and implication," American Journal of Plant Science, vol. 3, pp. 177-184, 2012.

[44] S. H. Wu, C. F. Hsieh, and M. Rejmánek, "Catalogue of the naturalized flora of Taiwan,” Taiwania, vol. 49, pp. 16-31, 2004.

[45] Q. Q. Huang, J. M. Wu, Y. Y. Bai, L. Zhou, and G. X. Wang, "Identifying the most noxious invasive plants in China: role of geographical origin, life form and means of introduction," Biodiversity and Conservation, vol. 18, no. 2, pp. 305-316, 2009.

[46] S. Tripathi and R. P. Shukla, "Effect of clipping and grazing on various vegetational parameters of grassland communities of Gorakhpur, Uttar Pradesh," Tropical Ecology, vol. 48, no. 1, pp. 61-70, 2007.

[47] K. S. Dogra, R. K. Kohli, and S. K. Sood, "An assessment and impact of three invasive species in the Sivalik Hills of 
Himanchal Pradesh, India," International Journal of Biodiversity Conservation, vol. 1, pp. 4-10, 2009.

[48] K. G. Saxena, "Biological invasion in the Indian sub-continent: review of invasion by plants," in Ecology of Biological Invasion in the Tropics, P. S. Ramakrishnan, Ed., pp. 53-73, International Scientific Publications, New Delhi, India, 1991.

[49] S. Tripathi, Plant diversity of grassland of north-eastern U.P. with emphasis on population of Parthenium hysterophorus L. [Ph.D. thesis], Gorakhpur University, Gorakhpur, India, 1999.

[50] S. K. Sood, S. Kumar, K. S. Dogra, and R. Sharma, "Alien plants distribution and ecology in the temple-courtyards of Himachal Pradesh, North-West Himalaya," Himachal Pradesh University Journal, pp. 1-11, 2011.

[51] C. H. Chou, "Allelopathic researches in the subtropical vegetation in Taiwan," Comparative Physiology and Ecology, vol. 5, pp. 222-234, 1980.

[52] P. S. Ramakrishnan, Ecology of Biological Invasions in the Tropics, International Scientific, New Delhi, India, 1991.

[53] K. G. Saxena and P. S. Ramakrishnan, "Partitioning of biomass and nutrients in the secondary successional herbaceous population subsequent to Slash and Burn," Proceedingsof the Indian National Science Academy, vol. 8, pp. 807-818, 1982. 

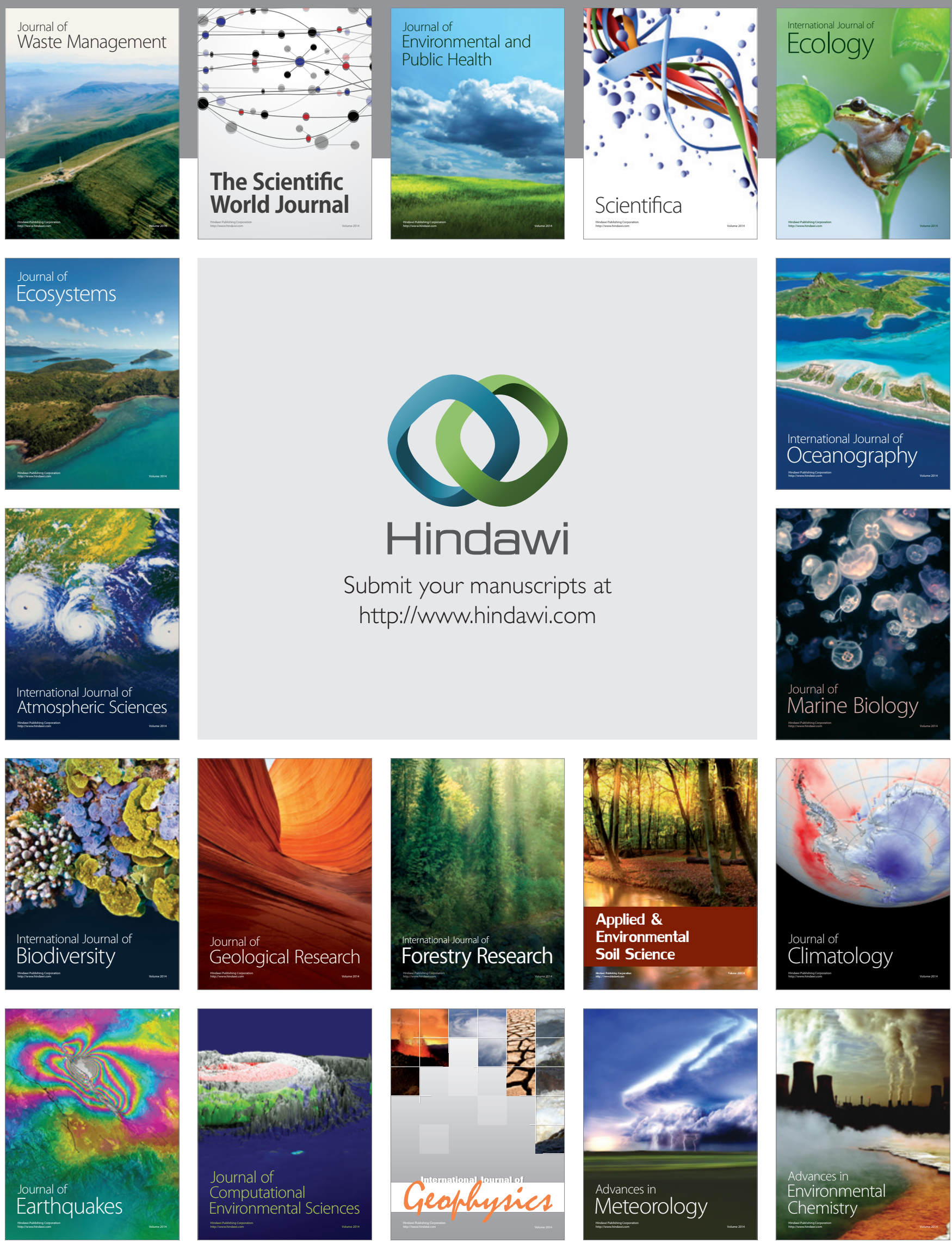